\title{
Dolphin Whistle Track Estimation Using Sequential Monte Carlo Probability Hypothesis Density Filter
}

\author{
Imtiaz Ahmed
}

Department of Applied Physics, Electronics and Communication Engineering, Dhaka University, Dhaka-1000, Bangladesh

(Received: 20 November 2012; Accepted: 16 September 2013)

\begin{abstract}
This article focuses on possible automation of dolphin whistle track estimation process within the context of Multiple Target Tracking (MTT). It provides automatic whistle track estimation from raw hydrophone measurements using the Sequential Monte Carlo Probability Hypothesis Density (SMC-PHD) filter. Hydrophone measurements for three different types of species namely bottlenose dolphin (Tursiops truncates), common dolphin (Delphinus delphis) and striped dolphin (Stenella coeruleoalba) have been used to benchmark the tracking performance of the SMC-PHD filter against three major challenges- the presence of multiple whistles, spontaneous death/birth of whistles and multiple whistles crossing each other. Quantitative analysis of the whistle track estimation accuracy is not possible since there is no ground truth type track for the dolphin whistles. Hence visual inspection of estimated tracks has been used corroborate the satisfactory tracking performance in the presence of all three challenges.
\end{abstract}

Keywords: Multiple Target Tracking, Probability Hypothesis Density Filter, Dolphin Whistle Tracking, Sequential Monte Carlo Method.

\section{Introduction}

Dolphin species identification and classification using acoustic techniques has been an active field of research since it supports conservation efforts ${ }^{1}$. These acoustic techniques, known as Passive Acoustic Monitoring (PAM) generally used to aid visual detection and classification of different species ${ }^{2}$. PAM uses raw acoustic recording of whistles from hydrophone measurements as they have been shown to contain species specific information ${ }^{3}$. Automatic extraction of these whistle tracks from the noisy measurements is of immense scientific interest since they can be used in species classification algorithms.

Most of the whistles tracking algorithms rely on spectrogram techniques ${ }^{4}$. A promising alternative to these conventional approaches can be the Probability Hypothesis Density (PHD) filter which jointly estimates the state and the number of targets from a set of noisy measurements ${ }^{5}$. Recently it has been shown that one variant of the PHD filter which is known as the Gaussian Mixture PHD (GMPHD) filter can produce accurate whistle tracks ${ }^{6}$. But in general, the recursion in PHD filter requires solving multidimensional integrals that do not, have closed-form solutions. As a result particle approximations are developed which is called the SMC-PHD filter ${ }^{7}$. The objective of this article is to automate the whistle track estimation process from the noisy hydrophone measurements using the SMCPHD filter. These reliable track estimates are absolutely essential for any algorithm for species classification, widely used in endangered Dolphin species conservation and mitigation efforts.

\section{Difficulties in Dolphin Whistle Frequency Tracking}

Performance of the species identification algorithms relies on the accuracy of the whistle track estimation process. The challenges in tracking the frequency contents of dolphin whistle can be attributed to: (i) death/birth of frequency tracks which means that number of whistles is unknown and generally varies over time (ii) presence of large amount of background noise in the recorded signal (iii) presence of multiple whistles cross each other. Based on challenges mentioned in (i) and (iii), estimation and tracking dolphin whistle can be regarded as MTT problem. In this work the potential of the SMC-PHD filter approach to tackle these challenges have been investigated.

\section{The SMC-PHD Filter}

In the MTT problems, targets evolve in the state space defined by the dynamical models. The local maxima of the PHD functions indicate the target positions in the space. At each time step the SMC-PHD approximates the target posterior PHD function by a weighted set of $N_{k}$ particles $\left\{x_{k}^{(i)}, w_{k}^{(i)}\right\}_{i=1}^{N_{k}}$.,8. The expected number of the targets is then given by the sum of the particle weights. State extraction from the group of particles is performed by a methods based on measurements. This allows the joint estimation of the number of targets and their states, from a set of noisy measurements.

In essence, the SMC-PHD filter algorithm propagates a set of particles through prediction and update stages. At first the filter predicts a set of particles at time instant $k$, both for persistent $\tilde{x}_{k \mid k-1, p}^{(i)}$ and newborn target $\tilde{x}_{k \mid k-1, b}^{(i)}$, drawn from the state transition density $f_{k \mid k-1}$. The filter will update the weights of the particles once the measurement $Z_{k}$ is available through likelihood function $\mathcal{L}\left(z_{k} \mid \cdot\right)$. One important point to note is that two different particle sets (persistent and newborn) $\left\{\widetilde{w}_{k \mid k, p}^{(i)}\right\}_{i=1}^{L_{k-1}}$ and $\left\{\widetilde{w}_{k \mid k, b}^{(i)}\right\}_{i=L_{k-1}+1}^{L_{k-1+J_{k}}}$ are resampled separately using stratified resampling ${ }^{\mathbf{9}, \mathbf{1 0}, \mathbf{1 1}}$. The resampling step is needed in order for the filter to avoid the problem of degeneracy as the number of the non-zero particle weights would otherwise become negligible after a few iterations. After resampling the number of particles for persistent target is maintained at a value of $\eta$ and the number of particles for newborn target is chosen to be $J_{k}$. These particles are approximate representation of the MT posterior density function. The location and the number of

*Author of Correspondence.email: imtiaz@univdhaka.edu 
local maxima of this posterior density indicate the target position and number respectively.

\section{State Space Model for Dolphin Whistle Tracking}

In MTT context, different dolphin whistles represents different targets. In this work state $x_{k}$ at time step $k$ consists of frequency $f$ and chirp rate $\alpha$ :

$$
x_{k}=\left[\begin{array}{ll}
f & \alpha
\end{array}\right]^{T}
$$

The chirp rate $\alpha$ defines the time rate of change of whistle frequency and has the unit $\mathrm{Hz} / \mathrm{s}$.

A linear Gaussian discrete state space model is used for dolphin whistle tracking:

$$
x_{k}=\left[\begin{array}{ll}
1 & T \\
0 & 1
\end{array}\right] x_{k-1}+v_{k}
$$

where $v_{k}$ is a zero mean Gaussian noise with a diagonal covariance matrix, so that system noise of the frequency and sweep rate are uncorrelated with different variances i.e. $\Sigma_{v}$, $f_{s}$ is the sampling frequency related with sampling period $T=\frac{1}{f_{s}}$. The state transition density $f_{k \mid k-1}\left(x_{k} \mid x_{k-1}\right)$ is $\mathcal{N}\left(x_{k} ; m_{f_{k \mid k-1}}, \Sigma_{f_{k \mid k-1}}\right)$ where $m_{f_{k \mid k-1}}=0_{1 \times 2}$ and $\Sigma_{f_{k \mid k-1}}=\left[\begin{array}{cc}\sigma_{f}^{2} & 0 \\ 0 & \sigma_{\alpha}^{2}\end{array}\right]$. Only frequency measurements are available, and the measurement model is

$$
z_{k}=H x_{k}+w_{k}
$$

where the measurement matrix $H$ is $H=\left[\begin{array}{ll}1 & 0\end{array}\right]$ and $w_{k}$ is zero mean Gaussian noise with covariance $\Sigma_{w}$. Hence the likelihood function $\mathcal{L}\left(z_{k} \mid x_{k}\right)$ is a Gaussian normal density $\mathcal{N}\left(x ; m_{\mathcal{L}}, \Sigma_{\mathcal{L}}\right)$ where $m_{\mathcal{L}}=0_{1 \times 1}$ and $\Sigma_{\mathcal{L}}=\Sigma_{w}$.

\section{Models for Spontaneous Birth of Whistle Frequency}

In general a-priori knowledge about where targets are likely to appear is commonly not available. Then a noninformative uniform prior can be use but this would require a large number of particles for reliable estimate of the state. This problem can be avoided by using the approach which allows targets to appear in the vicinity of measurements ${ }^{\mathbf{1 2}}$. As at each time scan there are multiple measurements available which may originate from either target or clutter. Hence if $Z_{k}$ denotes the measurement set at time scan $k$ then the intensity function $\gamma_{k}$ of the spontaneous birth PHD function is a Gaussian mixture:

$$
\gamma_{k}=\frac{1}{\left|Z_{k}\right|} \sum_{z \in Z_{k}} \mathcal{N}\left(x ;[z, 0], \Sigma_{v}\right)
$$

where $\left|Z_{k}\right|$ denotes the cardinality of the set $Z_{k}$. This model simulates the assumption that the whistle may appear spontaneously in the vicinity of measurements ${ }^{12}$.

\section{Measurement Generation Process}

Implementation of the SMC-PHD filter requires a set of measurements at discrete instants of time for joint estimation of the state and the target number. These measurements are generated from raw audio data from hydrophone using whistle contour detection based on a modified spectrogram which effectively reduces the effect of echolocation clicks and background noises ${ }^{13}$. Frequency measurements for three dolphin species namely bottlenose dolphin (Tursiops truncates), common dolphin (Delphinus delphis) and striped dolphin (Stenella coeruleoalba) have been generated using this modified spectrogram technique.

\section{Parameter Selection for the SMC-PHD Filter}

At the start of the processing filter needs to be initialized. This initialization step requires a number of particles to be drawn from a distribution for each of the targets. The number of target whistle frequency components is a random number initially. For each of these targets $\eta=10$ number of particles where drawn from the state transition density $\mathcal{N}\left(x_{k} ; 0_{1 \times 2}, \Sigma_{f_{k \mid k-1}}\right)$ where $\Sigma_{f_{k \mid k-1}}=\left[\begin{array}{cc}\sigma_{f}^{2} & 0 \\ 0 & \sigma_{\alpha}^{2}\end{array}\right]$. Experimentally the values of $\sigma_{f}$ and $\sigma_{\alpha}$ are found to be 10 and 100 respectively. A compromise between particle number and accuracy track detection is made by choosing $\eta=10$ and $\rho=10$. As particles are samples of state space, i.e. each particle is of the form $[f \alpha]^{T}$, two separate distributions are used for frequency and chirp rate. For the frequency $f$ the distribution is chosen to be uniformly distributed over the range $2 \mathrm{kHz}-30 \mathrm{kHz}$, i.e. $f \sim \mathcal{U}$ [2 kHz, $30 \mathrm{kHz}$ ]. This is because the fundamental frequency of the whistles may appear anywhere in that range with equal probability. The standard values for chirp rate $\alpha \sim \mathcal{U}[8 \mathrm{kHz} / \mathrm{s}, 60 \mathrm{kHz} / \mathrm{s}]$.

The target survival $p_{S}$ and the target detection probability $p_{D}$ are taken to be 0.99 and 0.95 . The clutter intensity $\kappa_{k}$ is assumed to be independent of time, i.e. $\kappa_{k}=\kappa$. It is assumed that the measurement generated using the modified spectrogram technique contains on the average $r=5$ clutter samples which are uniformly distributed over the frequency range of $2 \mathrm{kHz}-30 \mathrm{kHz}$ and hence $\kappa=\frac{5}{28000}$. The SMCPHD filter makes the decision about the presence of the target by comparing the weights of the particles to a threshold $W_{t h}=0.8$. Table- 1 summarizes all values of the simulation parameter used to implement the SMC-PHD filter.

\section{Simulation Results and Discussions}

The whistle track estimates produced by the SMC-PHD filter are superimposed on measurements for all three dolphin species and are shown in Fig.1-Fig.3. Automatic extraction of dolphin whistle tracks form the acoustic measurement is challenging due to different factors such as the presence of multiple whistles; multiple whistles crossing 
Table 1. Simulation Parameters used in Dolphin Whistle Tracking using the SMC-PHD Filter

\begin{tabular}{|c|c|}
\hline Simulation Parameter & $\begin{array}{l}\text { Simulation Parameter } \\
\text { Specification }\end{array}$ \\
\hline $\begin{array}{l}\text { Importance Sampling Density } \\
\text { For Persistent Target, } q(\cdot)\end{array}$ & $\mathcal{N}\left(x_{k} ; 0_{2 \times 1}, \Sigma_{v}\right)$ \\
\hline $\begin{array}{l}\text { Importance Sampling Density } \\
\text { For New Born Target, } p(\cdot)\end{array}$ & $\frac{1}{\left|Z_{k}\right|} \sum_{z \in Z_{k}} \mathcal{N}\left(x ;[z, 0], \Sigma_{v}\right)$ \\
\hline $\begin{array}{l}\text { Covariance of the State } \\
\text { Transition Density, } \Sigma_{v}\end{array}$ & {$\left[\begin{array}{cc}\sigma_{f}^{2} & 0 \\
0 & \sigma_{\alpha}^{2}\end{array}\right]$} \\
\hline $\begin{array}{lr}\text { Standard } & \text { Deviation of } \\
\text { Frequency } & \text { Measurement } \\
\text { Noise, } \sigma_{f} & \end{array}$ & 10 \\
\hline $\begin{array}{l}\text { Standard Deviation of Sweep } \\
\text { Rate Noise, } \sigma_{\alpha}\end{array}$ & 100 \\
\hline $\begin{array}{l}\text { Standard Deviation of } \\
\text { Measurement Noise, } \sigma_{w}\end{array}$ & 10 \\
\hline $\begin{array}{l}\text { Initial Probability Distribution } \\
\text { for frequency } f\end{array}$ & $f \sim \mathcal{U}[2 \mathrm{kHz}, 30 \mathrm{kHz}]$. \\
\hline $\begin{array}{l}\text { Initial Probability Distribution } \\
\text { for sweep rate } \alpha\end{array}$ & $\begin{array}{r}\alpha \sim \mathcal{U}[8 \mathrm{kHz} / \mathrm{s}, 60 \mathrm{kHz} \\
/ \mathrm{s}]\end{array}$ \\
\hline $\begin{array}{l}\text { Average Number of Clutter } \\
\text { Point per Scan, } r\end{array}$ & 5 \\
\hline Intensity of Poisson clutter, $\kappa$ & $\frac{5}{28000}$ \\
\hline Target Survival Probability, $p_{S}$ & 0.99 \\
\hline $\begin{array}{l}\text { Target Detection Probability, } \\
p_{D}\end{array}$ & 0.95 \\
\hline $\begin{array}{l}\text { Number of Particles per } \\
\text { Persistent Target, } \eta\end{array}$ & 10 \\
\hline $\begin{array}{l}\text { Number of Particles New-born } \\
\text { Target per Measurement, } \rho\end{array}$ & 10 \\
\hline Threshold, $W_{t h}$ & 0.8 \\
\hline Sampling Period, $T$ & 1 \\
\hline
\end{tabular}

each other and number of whistles is varying over time. A close examination on the simulation results presented in Fig.1 to Fig.3 reveals the fact that the SMC-PHD filter has successfully produced whistle track estimates from a set of noisy measurements for all three different dolphin species. The SMC-PHD filter has tracked multiple whistles discarding clutters even when they cross each other as depicted in Fig.2 and Fig.3. At each time step the filter calculated the expected number of whistles and hence can cope up with the time varying number of whistles. It also ensures that the tracks are automatically initiated and terminated. These results suggest that the automation in dolphin whistle tracking has been achieved using the SMCPHD filter.

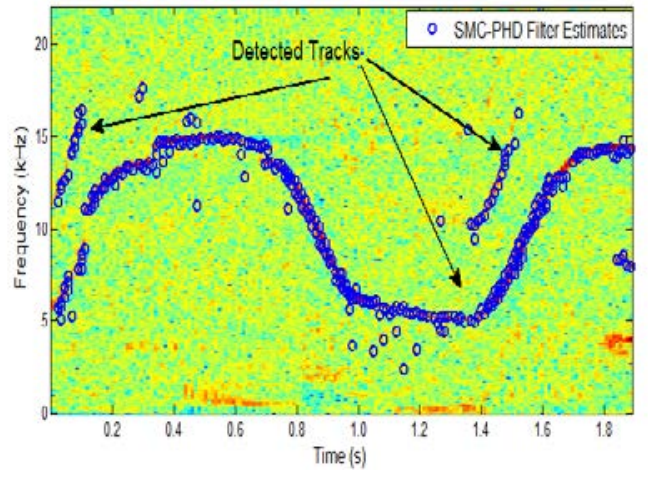

Fig. 1. Estimated whistle tracks from the SMC-PHD filter superimposed on spectrogram for bottlenose dolphin (Tursiops truncates).

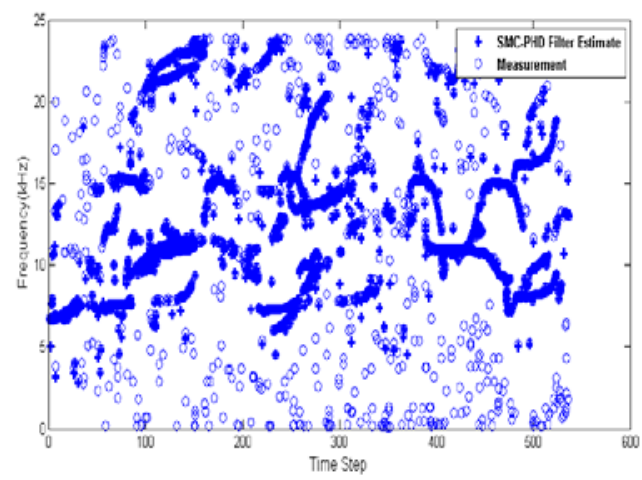

Fig. 2. Estimated whistle tracks from the SMC-PHD filter superimposed on measurements for common dolphin (Delphinus delphis).

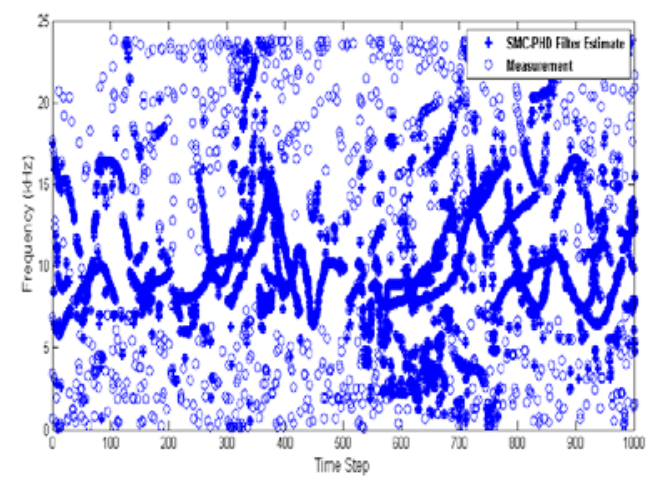

Fig. 3. Estimated whistle tracks from the SMC-PHD filter superimposed on measurements for striped dolphin (Stenella coeruleoalba).

\section{Conclusions}

This work has investigated the possibility of dolphin whistle track automation in the context MTT. It has been demonstrated that satisfactory track estimates can be produced automatically using the SMC-PHD filter. Simulation results suggest that the SMC-PHD filter has successfully produced reliable track estimates discarding significant clutter points for all three species in the presence of multiple whistles, spontaneous death/birth of whistles and multiple whistles crossing each other. In future these accurate track estimates produced by the SMC-PHD filter can be combined with different dolphin species identification 
algorithms to build a complete system. The complete system will identify and classify different dolphin species by processing raw acoustic recordings obtained from hydrophones. Hence a complete dolphin species identification system based on PAM can be realized.

\section{References}

1. Oswald, J. N., J. Barlow and T. F. Norris, 2003. Acoustic Identification of Nine Delphinid Species in the Eastern Tropical Pacific Ocean, Marine Mammal Sci., 19(1), 20-37.

2. Oswald, J. N., S. Rankin, J. Barlow and M. O. Lammers, 2007. A Tool for Real-Time Acoustic Species Identification of Delphinid Whistles, Journal of the Acoust. Soc. Am., 122(1), 587-595.

3. Steiner, W. W., 1981. Species-Specific Differences in Pure Tonal Whistle Vocalizations of Five Western North Atlantic Dolphin Species, Behavioral Ecology and Sociobiology, 9(4), 241-246.

4. Lampert, T. A. and S. E. M. O’Keefe, 2010. A Survey of Spectrogram Track Detection Algorithms, Applied Acoustics, 71 (2), 87-100.

5. Mahler, R., 2003. Multi-Target Bayes Filtering via FirstOrder Multi-Target Moments, IEEE Trans. on AES., 39(4), 1152-1178.

6. Ahmed, I., 2012. Dolphin Whistle Frequency Estimation using Gaussian Mixture Probability Hypothesis Density Filter, Int. J. Computer Vision and Signal Processing, 1(1), 9-14.
7. Vo, B. N., S. Singh and A. Doucet, 2005. Sequential Monte Carlo Methods for Multi-Target Filtering with Random Finite Sets, IEEE Trans. on AES., 41 (4), 1224-1245.

8. Ristic, B., D. E. Clark and B. N. Vo, 2010. Improved SMC implementation of the PHD filters, Conf. on Information Fusion (FUSION), 1-8.

9. Arulampalam, M. S., S. Maskell, J. Gordon and T. Clapp, 2002. A Tutorial on Particle Filters for online NonLinear/Non-Gaussian Bayesian Tracking, IEEE Trans. on SP., 50 (2), 174-188.

10. Clark, D.E., J. Bell, 2007. Multi-target State Estimation and Track Continuity for the Particle PHD filter, IEEE Trans. on AES, 43(4), 1441-1453.

11. Mahler, R., 2007. PHD Filters of Higher Order in Target Number, IEEE Trans. on AES, 43(4), 1523-1543.

12. Maggio, E., M. Taj and A. Cavallaro, 2008. Efficient MultiTarget Visual Tracking Using Random Finite Sets, IEEE Trans. on Circuits and Systems for Video Tech., 18(8), 10161027.

13. Gillespie, D., P. R. White, M. Caillat and J. Gordon, 2011, Development and Implementation of Automatic Classification of Odontocetes within PAMGUARD, Fifth International Workshop on Detection, Classification, Localization, and Density Estimation of Marine Mammals using Passive Acoustics, Timberline Lodge, Mt. Hood, Oregon, USA. 